\title{
Die FMH für den Tiers garant
}

\author{
Aus Sicht der Ärzteschaft ist im ambulanten Bereich (KVG) der Tiers garant die be- \\ vorzugte Abrechnungsmethode. Sie ist für alle beteiligten Akteure - Patient, Arzt \\ und Versicherer - prädestiniert, die Anforderung in Bezug auf Qualität, Kosten und \\ Effizienz unseres Gesundheitssystems zu erfüllen und weiter zu fördern.
}

\author{
Ernst Gähler ${ }^{a}$, \\ Irène Marty ${ }^{b}$, \\ Kerstin Schutz \\ a Dr. med., Vizepräsident FMH, \\ Verantwortlicher Ressort \\ Ambulante Tarife und \\ Verträge Schweiz \\ b FMH, Stv. Leiterin Ressort \\ Ambulante Tarife und \\ Verträge Schweiz \\ c FMH, Ressort Ambulante \\ Tarife und Verträge Schweiz
}

Korrespondenz:

FMH / Ressort Ambulante Tarife und Verträge Schweiz

Froburgstrasse 15

CH-4600 Olten

Tel. 0313591230

Fax 0313591238

tarife.ambulant[at]fmh.ch
Die FMH befürwortet die gemäss Art. 42 Abs. 1 KVG geltende Regel, dass im ambulanten Bereich grundsätzlich im Tiers garant abgerechnet wird, sofern zwischen Leistungserbringer und Versicherer nichts anderes vereinbart wurde.

Im folgenden Artikel sind Auszüge aus dem Positionspapier der FMH abgedruckt. Das gesamte Positionspapier finden Sie unter www.fmh.ch

\section{Ausgangslage}

Grundsätzlich existieren in der Schweiz im ambulanten Bereich zwei Abrechnungssysteme der Kostenvergütung in der Obligatorischen Krankenpflegeversicherung (OKP) [1].

\section{Tiers garant}

Haben Leistungserbringer und Kostenträger nichts anderes vereinbart, so gilt gemäss Art. 42 Abs. 1 KVG dieses System als Regelfall. Beim Tiers garant schuldet der Versicherte dem Leistungserbringer die Vergütung der Leistung. Die Versicherten haben in diesem Fall einen Anspruch auf eine Rückerstattung durch die Versicherung.

\section{Tiers payant}

Gemäss Art. 42 Abs. 2 KVG können aber Versicherer und Leistungserbringer vereinbaren, dass der Versicherer die Vergütung schuldet. Dieses System heisst Tiers payant.

Diese zwei Vergütungsarten Tiers garant und Tiers payant sind im KVG im Art. 42 geregelt [2]. Grossmehrheitlich wird in der Schweiz nach dem System des Tiers garant abgerechnet, wie es auch im KVG als vorherrschendes System genannt wird.

In den letzten Jahren wird vonseiten einzelner Krankenversicherer zunehmend Druck betreffend das Abrechnungssystem ausgeübt. Sie argumentieren, dass mit dem System Tiers payant, insbesondere durch die Einführung des elektronischen Datenaustausches (eDA) über einen Intermediär, prämienwirksam Kosten gespart werden können.

Seit geraumer Zeit bestehen daher erhebliche Differenzen zwischen den Versicherern und der Ärzteschaft über die Abwicklung des eDA, da via die ärzteeigenen TrustCenter im Tiers garant abgerechnet wird.
Über den Intermediär MediData, der mehrheitlich von den Versicherern betrieben und finanziert wird, wird hingegen über den Tiers payant abgerechnet.

Es ist verständlich, dass die Versicherer den Tiers payant bevorzugen: Mit steigendem Umsatz verändert sich das Verhältnis von Umsatz und Verwaltungskosten. Diese Relativierung ist seitens der Krankenversicherungen nicht unerwünscht.

\section{Gründe für den Tiers garant}

Im Tiers payant steigen das Abrechnungsvolumen und die Verwaltungskosten - diese Kostensteigerung könnte prämienwirksam werden.

Im reinen System des Tiers payant gehen alle Arztrechnungen automatisch und direkt zu den Krankenversicherungen. Aus Sicht der Ärzteschaft ist das ein volkswirtschaftlicher Unsinn, denn:

- Wie wir aus verschiedenen Untersuchungen wissen, ist der Anteil von Rechnungen mit kleinen Beträgen (kleiner als $100 \mathrm{CHF}$ ) in Arztpraxen im OKP-Bereich beachtlich. Diese werden sehr häufig von den PatientInnen direkt und «out of pocket» bezahlt. Würden all diese kleinen Arztrechnungen im Tiers payant vergütet, führt das nur zu Mehrkosten und zu teuren administrativen Umtrieben ohne jeglichen medizinischen Nutzen. Die Kosten eines Wechsels von Tiers garant zu einem flächendeckenden Tiers payant würden sich schätzungsweise auf ca. CHF 750 Mio. belaufen.

- Und auch jeder Franken, welcher über den Betrag der Franchise hinausgeht, wird im Tiers payant von den Versicherungen bezahlt und wirkt sich damit direkt auf die Prämien aus. Untersuchungen mit santésuisse haben gezeigt, dass eine Differenz von bis zu $15 \%$ zwischen den gestellten Rechnungen und den bei den Versicherungen zur Rückvergütung eingereichten Rechnungen besteht. Es ist also davon auszugehen, dass bei einem flächendeckenden System des Tiers payant die prämienwirksamen Kosten und im Speziellen die Verwaltungskosten deutlich steigen werden.

- Simulationen zeigen, würden alle «kleinen» Beträge den Krankenkassen eingereicht, könnte sich das Volumen eines Kantons um 5-8\% erhöhen, was eine Senkung des Taxpunktwertes in einem Kanton bewirken würde! 
So steigen die Verwaltungskosten und der Umsatz der Versicherer, ohne dass irgendein Mehrnutzen für die Versicherten generiert wird.

Im Tiers payant sind die Debitorenverluste sowie das Klumpenrisiko höher als im Tiers garant

Das Argument, dass die Zahlungsmoral beim System Tiers payant besser ist, kann anhand einer Auswertung der Ärztekasse exemplarisch anhand der Kantone St. Gallen und Zürich widerlegt werden.

Die Auswertungen der Ärztekasse in den beiden Kantonen Zürich und St. Gallen zeigen: Nach 30 Tagen ist der Anteil der bezahlten Rechnungen im Tiers payant kleiner als im Tiers garant. Auch die Zahlungsverluste sind in beiden Kantonen im Tiers garant tiefer!

Die Auswertungen über mehrere Kantone zeigen, dass die Debitorenverluste im Tiers garant generell

Abbildungen 1 und 2

Statistik Ärztekassen April 2011 bis März 2012 im Kanton Zürich und Kanton St. Gallen.

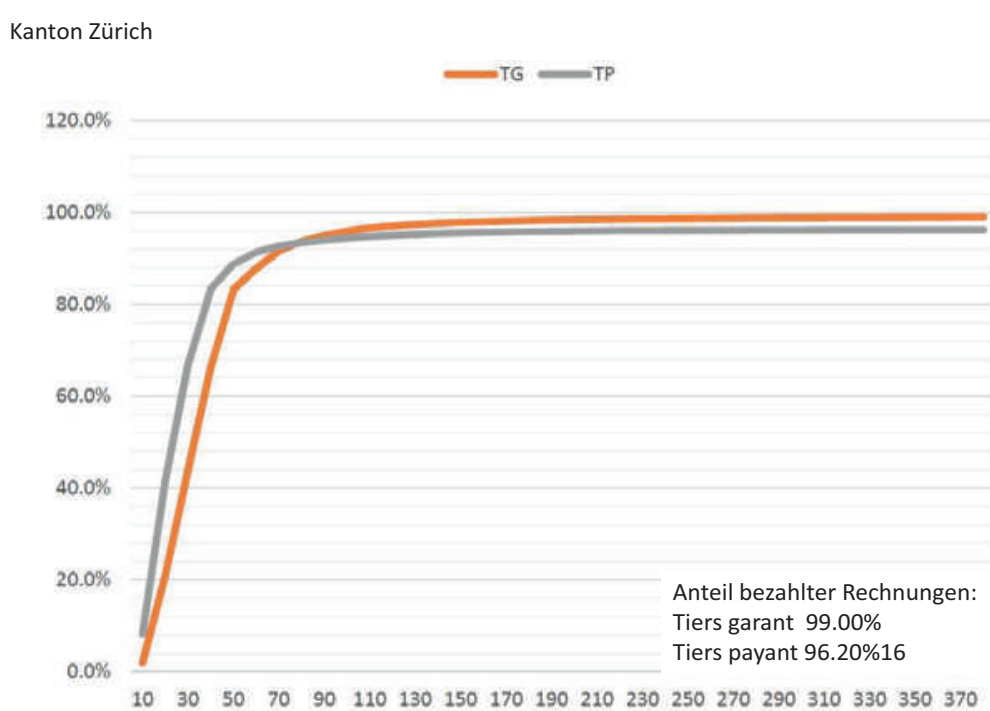

Kanton St. Gallen

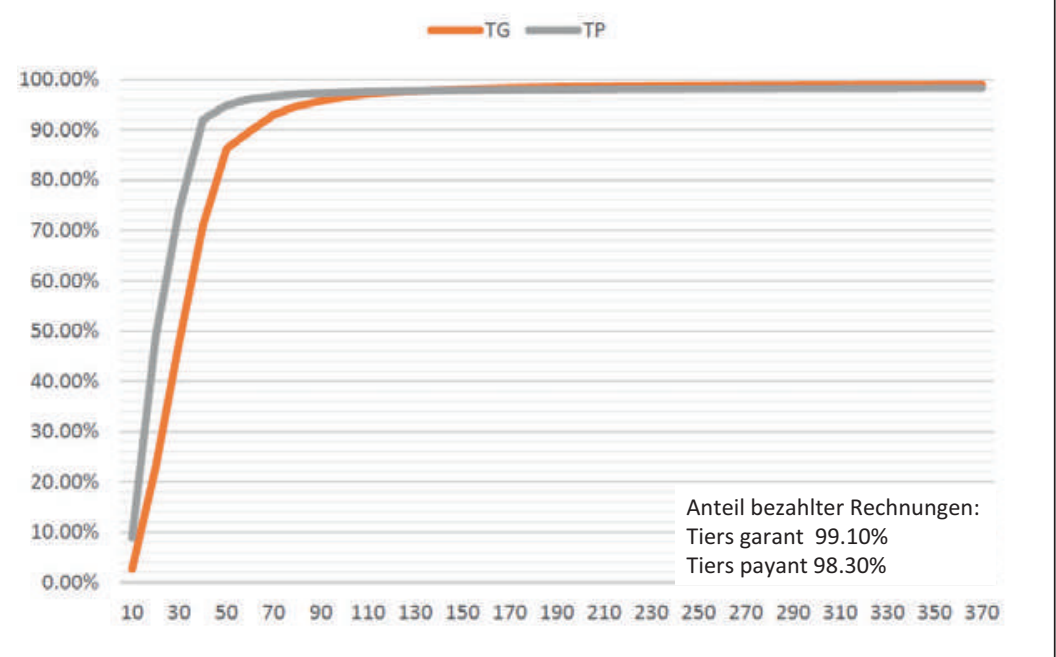

geringer ausfallen als im Tiers payant. Auch die Risikostreuung ist im Tiers garant grösser als im Tiers payant, da die Zahl der Schuldner (Patienten) natürlich grösser ist und der Betrag pro Schuldner kleiner.

Im Tiers payant gelten die Abtretungen pauschal, der Patient verliert die Entscheidungshoheit Im Tiers garant können im Einzelfall Abtretungserklärungen sinnvoll sein. Wenn abzusehen ist, dass eine Behandlung langwierig und kostenintensiv sein wird, kann der Arzt mit dem Patienten eine Abtretungserklärung [3] für einen definierten Zeitraum vereinbaren, um sich vor hohen Rechnungen zu schützen. Solche Abtretungserklärungen sollten jedoch individuell und situationsangepasst erfolgen. Im Tiers payant gäbe es diese «Abtretung» aber pauschal über alle Patienten und für alle Rechnungen. Der Patient verliert damit die Entscheidungshoheit, und der Datenschutz ist nicht mehr gewährleistet.

Die Kontrolle der Rechnung wird im Tiers payant den Versicherungen überlassen, die Möglichkeit der Einflussnahme des Patienten wird minimiert Der vom Bund gewünschte Anreiz zur Kosten- und Qualitätskontrolle durch den Patienten / die Patientin geht im Tiers payant verloren. Niemand kann eine Arztrechnung besser kontrollieren als die Patienten selber. Eine Umfrage von santésuisse hat wohl entgegen ihren Erwartungen - ergeben, dass 70\% der Patienten ihre Arztrechnungen kontrollieren.

Nicht zu vergessen ist aber auch der Vorteil, dass alle Ärztinnen und Ärzte, die im System des Tiers garant abrechnen, ihren Patientinnen und Patienten gegenüber so volle Transparenz gewähren.

\section{Datenschutz und Schutz vor dem «gläsernen} Patienten» durch den Tiers payant gefährdet Im Tiers payant wird jede Diagnose und Erkrankung von Patienten bei den Versicherern ersichtlich, weil jede Rechnung an den Versicherer geht.

Durch den äusserst filigranen und differenzierten TARMED-Tarif lässt sich problemlos die Diagnose und damit auch die Erkrankung und sogar eine Prognose über den Gesundheitszustand des Patienten / der Patientin ableiten. Das Datenschutzproblem wurde mehrfach vom eidgenössischen Datenschützer in seinen Berichten moniert, und er empfiehlt die Abwicklung der Arztrechnungen im Tiers garant über ein TrustCenter.

\section{Deshalb: Tiers garant und elektronischen Datenaustausch (eDA) über die TrustCenter wählen}

Jährlich gehen bei den TrustCentern 19 Mio. Rechnungen ein - insgesamt sind über eine Milliarde Datensätze in diesen ärzteeigenen TrustCentern gespeichert. Damit ist und bleibt die günstigste Variante im eDA die Nutzung der in den TrustCentern daten- 
schutzkonform und nahezu kostenfrei bereitgestellten elektronischen Rechnungen. Die Rechnungen, die von Intermediären wie MediData den Versicherern zur Verfügung gestellt werden, generieren Kosten (ca. 2 CHF pro Rechnung) - ein finanzieller Aufwand für die Versicherer, den die Prämienzahler schliesslich berappen.

Im Interesse der gesamten Ärzteschaft ist es aus oben genannten Gründen absolut zentral, dass weiterhin grundsätzlich im Tiers garant abgerechnet wird. Der elektronische Datenaustausch (eDA) über die TrustCenter ist dabei das präferierte System, denn es ist datenschutzkonform und ist für alle Betroffene das kostengünstigste System. Die FMH setzt einen ihrer Schwerpunkte in den kommenden Jahren darauf, die Abrechnungsmodalitäten in den Trust Centern noch weiter zu optimieren.

\section{Referenzen}

1 Der Tiers soldant ist eine dritte Vergütungsart, die das KVG (Art. 42 Abs. 1 letzter Satz KVG) vorsieht. Der Tiers soldant ist dann gegeben, wenn der Patient im Tiers garant seinen Rückerstattungsanspruch (d.h. seinen Nettoanspruch, also Rechnungsbetrag nach Abzug von Franchise und Selbstbehalt) gegenüber der Krankenkasse dem Arzt schriftlich abtritt.

2 Art. 42 KVG:

${ }^{1}$ Haben Versicherer und Leistungserbringer nichts anderes vereinbart, so schulden die Versicherten den Leistungserbringern die Vergütung der Leistung.

Die Versicherten haben in diesem Fall gegenüber dem Versicherer einen Anspruch auf Rückerstattung (System des Tiers garant). In Abweichung von Artikel 22 Absatz 1 ATSG2 kann dieser Anspruch dem Leistungserbringer abgetreten werden (Tiers soldant [Anm.d. A.]).

${ }^{2}$ Versicherer und Leistungserbringer können vereinbaren, dass der Versicherer die Vergütung schuldet (System des Tiers payant). Im Falle der stationären Behandlung schuldet der Versicherer, in Abweichung von Absatz 1, den auf ihn entfallenden Anteil an der Vergütung.

3 Art. 42 Abs. 1 letzter Satz KVG: In Abweichung von Art. 22 Absatz 1 ATSG kann dieser Anspruch dem Leistungserbringer abgetreten werden.

\title{
Wissen, was läuft.
} Das News-Paket der FMH.

\author{
Schweizerische Ärztezeitung, Today's Press, \\ FMH-Flash. Für Mitglieder kostenlos.
}

